\title{
Estudio exploratorio con futuras maestras sobre lenguas matemáticas para enseñar la relación entre área y volumen
}

\author{
Abigail Caro, Universitat Autònoma de Barcelona (España) \\ Núria Planas, Universitat Autònoma de Barcelona (España)
}

\begin{abstract}
Resumen
En este artículo resumimos un estudio exploratorio sobre el conocimiento de contenidos de la materia de educación matemática en un entorno de formación inicial de profesorado; desde nuestro posicionamiento sociocultural, nos fijamos en el papel mediador de las lenguas matemáticas utilizadas en la instrucción y en los materiales curriculares, y escogemos contenidos relativos a lenguas matemáticamente relevantes para la enseñanza y el aprendizaje de la relación entre área y volumen. Con base en respuestas a un cuestionario, primero examinamos qué saben o anticipan las futuras maestras acerca de la función de la lengua en la enseñanza y el aprendizaje del contenido matemático específico. Luego amplificamos el análisis con entrevistas selectivas guiadas por respuestas al cuestionario. Los resultados muestran estudiantes con una comprensión débil de contenidos de la materia que habrán de enseñar -conceptos de área y volumen-, a la vez que iniciándose en la construcción de contenidos sobre cómo enseñar la materia -lenguas matemáticas para enseñar la relación entre área y volumen.
\end{abstract}

Palabras clave. Aproximación sociocultural; aprender a enseñar matemáticas; formación inicial del profesorado de matemáticas; relación área-volumen; lengua como recurso.

Exploratory study with preservice primary teachers on mathematical languages for teaching the relationship between area and volume

Abstract

In this article, we report an exploratory study about the knowledge of mathematics education subject content in a setting of education preservice training; within our sociocultural positioning, we focus on the mediating role of mathematical languages used in instruction and curricular material, and choose content relative to mathematically-relevant languages for the teaching and learning of the relationship between area and volume. Based on responses from a questionnaire, we first examine what primary school student teachers know or anticipate of the function of language when teaching and learning the specific mathematical content. We then amplify the analysis by means of selective interviews guided by responses to the questionnaire. Our results show student teachers with weak understanding of contents of the subject to be taught-concepts of area and volume-while becoming initiated in the construction of contents on how to teach the subject-mathematical languages for teaching the area-volume relationship.

Keywords. Sociocultural approach; learning to teach mathematics; mathematics teacher preservice education; relationship between area and volume; language as resource.

\section{Introducción}

En la investigación en educación matemática y en particular en la línea sobre formación del profesorado, empiezan a ser abundantes los estudios sobre desarrollo profesional del profesorado en cuestiones de lengua y matemáticas (e.g. Planas, 2021; Prediger, 2019; Prediger, Erath \& Moser Opitz, 2019). No obstante, la investigación y la práctica sobre estas cuestiones siguen siendo escasas en los módulos de preparación inicial del profesorado de matemáticas de distintos niveles educativos (con algunas excepciones como el trabajo experimental en Noruega liderado por Rangnes y Meaney, 2021). Habiendo estudiado la lengua del profesor de matemáticas en la enseñanza (e.g. Planas, Badillo, \& Chico, 2019; Planas, Chico, García-Honrado, \& Arnal-Bailera, 2020), ahora nos preparamos para diseñar y experimentar acciones formativas sobre 
lenguas matemáticas para la enseñanza con estudiantes del Grado de Educación Primaria de la Universitat Autònoma de Barcelona. Se tomaron datos de cuestionario en febrero de 2020 en el contexto del Trabajo de Fin de Master de la primera autora (Caro, 2020), y la segunda autora realizó entrevistas en octubre de 2020 con un grupo reducido de estudiantes de ese Grado que habían respondido al cuestionario el curso anterior.

Por una parte, tomamos los estudios clásicos de Shulman $(1986,1987)$ sobre el conocimiento profesional para la enseñanza y las relaciones entre conocimiento pedagógico y conocimiento de la disciplina. Por otra parte, nos inspiran estudios con orientaciones socioculturales sobre lengua y educación matemática (Planas, Morgan \& Schütte, 2018, 2021; Radford \& Barwell, 2016), particularmente los que vislumbran la lengua como contenido esencial -no optativo ni aislado- de enseñanza y aprendizaje en la formación inicial y continua del profesorado de matemáticas (Erath, Ingram, Moschkovich, \& Prediger, 2021; Essien, Chitera, \& Planas, 2016). Desde nuestra perspectiva, si comprendemos cómo formar al profesorado en el uso particular de las lenguas matemáticas para enseñar y aprender contenidos matemáticos específicos y si asumimos que parte de esta formación acabará siendo representada en la práctica de aula, nos estaremos acercando a una enseñanza con mayor impacto en el aprendizaje. Somos conscientes de que no se ha logrado establecer una relación directa entre el conocimiento teórico del profesorado y sus prácticas de enseñanza; esto ocurre en parte porque la generación de conocimiento se gesta en un contexto que a menudo es distinto al contexto en el que se gesta la práctica de enseñanza. Aun sabiendo esto, adoptamos una aproximación sociocultural a la formación del profesorado de matemáticas (White, Crespo, \& Civil, 2016) donde teoría y práctica se encuentran en el espacio común que los participantes llegan a construir, con los instrumentos e ideas que se les han facilitado, para reflexionar sobre su práctica profesional y anticipar acciones de enseñanza.

\section{La lengua como contenido en la formación del profesorado de matemáticas}

Formar al profesorado de matemáticas implica apoyar la construcción de conocimientos matemáticos suficientes y de conocimientos pedagógicos generales de gestión y de organización del aula, además de apoyar la construcción de conocimientos pedagógicos para enseñar matemáticas. El trabajo del psicólogo estadounidense Lee S. Shulman es pionero en la discusión del conocimiento que los profesores han de tener del contenido que enseñan. Mientras que en Shulman (1986a, 1986b) se introdujo el conocimiento del contenido, el conocimiento didáctico del contenido y el curricular, en Shulman (1987) se avanzó en la creación y el desarrollo de siete categorías de conocimiento: 1) pedagógico general; 2) pedagógico específico del contenido; 3 ) de los alumnos y de sus características; 4) de los contextos educativos; 5) de los fines, valores y sustentos histórico-filosóficos educativos; 6) del contenido; 7) del currículo.

Dos décadas más tarde, Ball, Thames y Phelps (2008) propusieron un modelo de conocimiento para enseñar matemáticas (popularizado como MKT por Mathematical Knowledge for Teaching), que es una revisión creativa de las categorías de Shulman con los ejes del conocimiento matemático y del conocimiento didáctico del contenido matemático. Si bien en Shulman (1987) se menciona el papel del conocimiento de las culturas implicadas en las situaciones de enseñanza y aprendizaje y en Shulman (2005, p. 53) incluso se pone el ejemplo de un profesor universitario que enseña pidiendo a los estudiantes que presten atención a la lengua utilizada en los materiales de clase, son consideraciones generales y el ejemplo se elabora para el caso de la formación inicial de abogados en una escuela de leyes. Ball et al. (2008) sí mencionan específicamente el conocimiento pedagógico de la lengua para la enseñanza de matemáticas. En ese artículo 
se resalta la tarea didáctica de problematizar ciertos usos de la notación y de las lenguas matemáticas en contextos de formación de profesorado (p. 10), junto con esta otra mención relativa al papel de la lengua en la enseñanza de matemáticas:

[Los profesores de matemáticas] necesitan ser capaces de hablar explícitamente sobre cómo se utiliza la lengua matemática (e.g. cómo el significado matemático de 'arista' difiere de la referencia cotidiana a la arista de una mesa). (nuestra traducción, p. 11)

A pesar de que nuestro interés no es categorizar el conocimiento del uso de la lengua para enseñar y aprender matemáticas, sí vemos relevante señalar su ubicación dentro del conocimiento didáctico del contenido matemático. Esto implica que modelos altamente referenciados en la investigación sobre formación de profesorado de matemáticas incluyen categorías que avalan la consideración de aspectos de conocimiento de la cultura y de la lengua en relación con contenidos matemáticos específicos. Este hecho contrasta con la poca atención al recurso de la lengua en entornos de formación de profesorado de matemáticas, a diferencia de lo que ocurre por ejemplo con la atención al uso didáctico de recursos tales como los libros de texto o las tecnologías digitales.

Hay generalidades acerca del uso pedagógico de la lengua en el aula que el profesor de cualquier materia debe conocer tales como incorporar preguntas que fomenten la participación o que activen ciertos patrones de interacción (Ingram, 2018). Sin embargo, el profesorado de matemáticas debe además aprender una lengua experta de carácter profesional para la enseñanza de la matemática escolar, cuyas formas y funciones apoyen la producción y comprensión de significados matemáticos concretos (Chitera, 2011; Planas, 2018, 2021). Al respecto, los avances en la conceptualización de la lengua como recurso orientado a enseñar y aprender matemáticas (Planas, Morgan y Schütte, 2018) sugieren enseñar de manera integrada cuestiones lingüísticas (de vocabulario y de sintaxis propios del registro matemático especializado) y discursivas (de puesta en práctica de ese vocabulario y de esa sintaxis en la resolución de tareas matemáticamente interesantes en contextos de clase). En su formación inicial y por muy obvio que parezca, el profesorado de matemáticas necesita entender que las palabras "área" y "volumen", por ejemplo, no tienen una relación fija ni obvia con los significados que se les asocian en la matemática escolar, por lo que estos significados deben ser objeto de enseñanza explícita mediante una lengua experta que explique con claridad y precisión los conceptos, sus cualidades y sus relaciones entre ellos y con otros conceptos.

\section{La relación entre área y volumen como contenido curricular}

En nuestro estudio trabajamos con dos contextos curriculares locales: el currículo de matemáticas para la educación primaria en Catalunya y el currículo de educación nmatemática para la formación inicial de profesorado en el Grado de Educación Primaria de la Universitat Autònoma de Barcelona. En ambos currículos se considera el contenido matemático "relación entre área y volumen", pero con variaciones en los significados que se asocian mediante ejemplos de tareas. De la lectura de las guías y programas docentes universitarios y del documento curricular para la etapa de primaria, se observa que los significados para este contenido matemático no deben suponerse compartidos entre formadores de profesorado ni entre profesorado, dado que por ejemplo no se escribe explícitamente acerca del significado de área de un cuerpo con volumen. Precisamente Clements et al. (2018) documentan el fenómeno más global de trabajar la noción de área sobre todo vinculada a la geometría plana en las primeras edades escolares e incluso en etapas posteriores; como consecuencia, esta noción se acostumbra a asociar a figuras bidimensionales y no se conecta con el área de cuerpos con volumen. 
Por un lado, la administración educativa catalana establece la dimensión de conexiones en el currículo de matemáticas para educación primaria con mención a relaciones entre conceptos y entre significados de un concepto (Departament d'Educació, 2013). Con los conceptos de área y volumen, el aprendizaje de conexiones no es trivial para el alumnado de primaria, tal como indica la producción escrita de un alumno de segundo curso de primaria en una tarea con dos cilindros en De Gamboa, Badillo y Ribeiro (2015). Por otro lado, el currículo de educación matemática para futuras maestras del Grado de Educación Primaria de nuestra Universidad incluye el trabajo de la relación entre área y volumen, junto con el de la relación entre perímetro y área. Este currículo establece que las futuras maestras deben aprender a identificar un concepto básico en situaciones donde tenga distintos significados; para la noción de área y el trabajo de significados matemáticos que se mantienen invariantes en las geometrías plana y espacial, se acostumbran a proponer tareas como la de la Figura 1 (Planas, 2006).

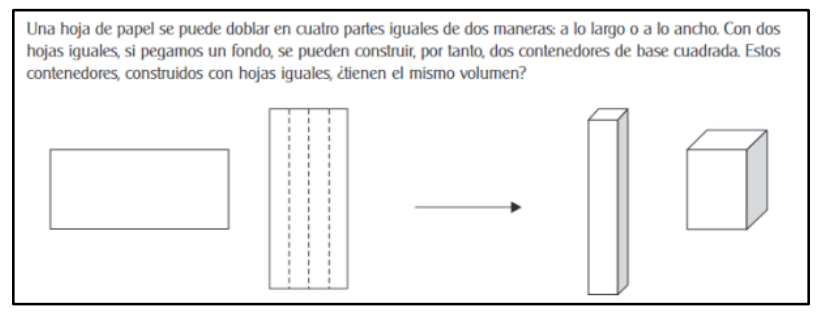

Figura 1. Tarea sobre la relación entre área y volumen

En nuestro contexto institucional, cabe notar lo común de encontrar lenguas matemáticas poco precisas en torno al trabajo de las nociones de área y de volumen. Por ejemplo, en las denominadas Pruebas de Aptitud Personal para el acceso a los Grados en Educación Infantil y en Educación Primaria, en 2018 un enunciado asemejaba la noción de volumen a la de medida del volumen: “¿Cuál es el volumen de la figura que se muestra a continuación [se representa en la hoja un sólido formado por cinco cubos] teniendo en cuenta que los lados de cada cubo hacen $2 \mathrm{~cm}$ ?" Hemos traducido el texto del catalán lo más literalmente posible por lo que es intencionado mantener el verbo "hacen". Hemos encontrado este uso poco preciso y ambiguo de lenguas matemáticas en otros contextos institucionales y académicos. En cualquier caso, las participantes de nuestra investigación fueron admitidas al Grado de Educación tras enfrentarse a las Pruebas mencionadas y superarlas, lo cual en concreto implica haber comprendido y respondido a lo que se pedía en los enunciados de los problemas de un modo aceptable para los correctores y para los diseñadores de los criterios de corrección.

\section{Pregunta, participantes y métodos}

La pregunta que nos guía es la siguiente: ¿Cuáles son los indicios de conocimiento de futuras maestras de primaria sobre las lenguas matemáticas para la enseñanza de la relación entre área y volumen? De acuerdo con el marco teórico adoptado, donde palabras y enunciados no tienen significados matemáticos fijos ni obvios, sino que la construcción de esos significados depende del uso de palabras y enunciados en un entorno de práctica concreto, miramos la pregunta desde la perspectiva de cómo las futuras maestras construyen sus significados para la relación entre área y volumen en los entornos de uso de la lengua escrita y hablada desarrollados para la investigación. Así como los significados son situados y la lengua en uso es una de sus representaciones, nuestros resultados serán por tanto relativos a los entornos creados, a sus características y a las formas de representación involucradas (Gee, 1999). La cantidad de datos, el carácter exploratorio de los análisis y la naturaleza situada y representativa de los 
resultados deben leerse según la intención última de provocar discusión y reflexión sobre la inclusión de contenidos de lengua en la formación de profesorado de matemáticas.

Nuestro objetivo es detectar conocimiento especializado del profesorado de matemáticas sobre lenguas expertas para la enseñanza de la relación entre área y volumen en la escuela. El logro parcial de este objetivo con datos de respuestas escritas al cuestionario se corresponde con resultados publicados en Caro (2020). El logro más avanzado del objetivo con datos de entrevista es posterior y supone una ampliación que refina interpretaciones de las futuras maestras acerca del potencial y uso didáctico de la lengua en la enseñanza de matemáticas. El enfoque es interpretativo en la consecución progresiva del objetivo. Combinamos métodos deductivos e inductivos de análisis del discurso escrito en respuestas de cuestionario y métodos inductivos de análisis del discurso hablado en entrevistas individuales basadas en una selección de respuestas al cuestionario y en una selección de datos ilustrativos de aulas de primaria.

El primer grupo participante fueron 51 estudiantes de segundo curso del Grado de Educación Primaria que en febrero de 2020 empezaban su primera asignatura de educación matemática, 'Aprendizaje de las Matemáticas y Currículum'. La recogida de datos se realizó mediante un cuestionario individual, creado para el estudio, publicado en Caro (2020) y respondido en el aula universitaria durante unos 45 minutos. Para asegurar que las tareas y su redactado fueran comprensibles, hubo un cuestionario algo distinto que se pilotó y que, tras introducir algunos cambios, dio lugar al cuestionario definitivo. La finalidad era facilitar la recolección de datos que nos facilitaran el acceso a significados que las futuras maestras producirían en la interpretación de conocimiento especializado para enseñar el contenido escolar de la relación entre área y volumen.

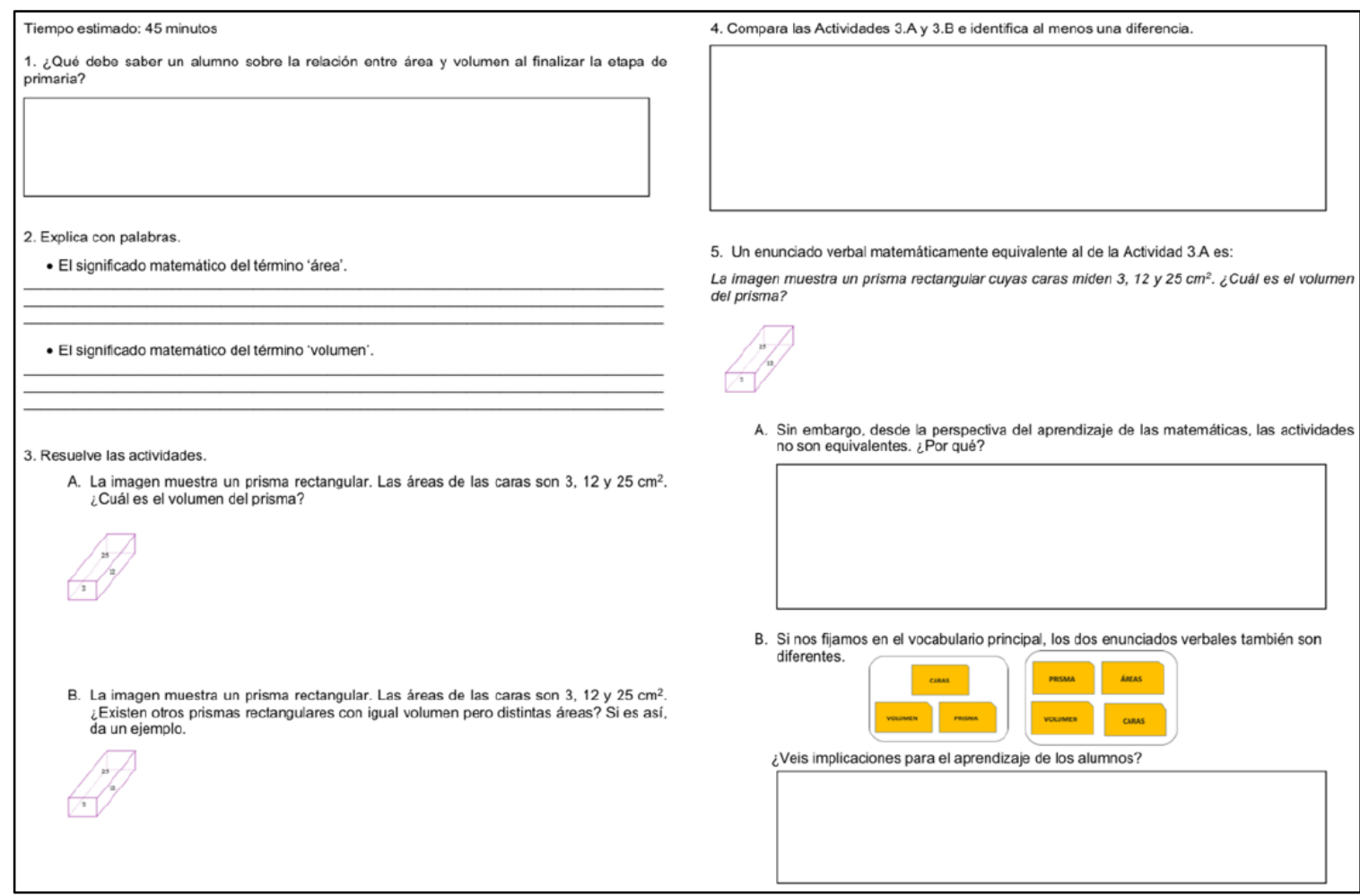

Figura 2. Ítems de las dos primeras páginas del cuestionario (continúa)

Las Figuras 2 y 3 dan idea de cómo alternamos tareas matemáticas (e.g. "Explica con palabras el significado matemático del término área"), didácticas (e.g. "Compara las Actividades $3 \mathrm{~A}$ y $3 \mathrm{~B}$ e identifica al menos una diferencia) y lingüísticas (e.g. "Si nos 
fijamos en las frases que hacen que los dos enunciados sean diferentes..."). Se optó por tareas donde las futuras maestras tuvieran que escoger y comunicar conocimientos matemáticos y razones acerca de las similitudes y diferencias, con respecto al aprendizaje matemático del alumnado de primaria, entre problemas matemáticos con aparentemente cambios menores en la redacción del enunciado. Las respuestas se trataron con anonimato y se numeraron de un modo que permitió rastrear las futuras maestras que más tarde se seleccionarían para las entrevistas individuales.

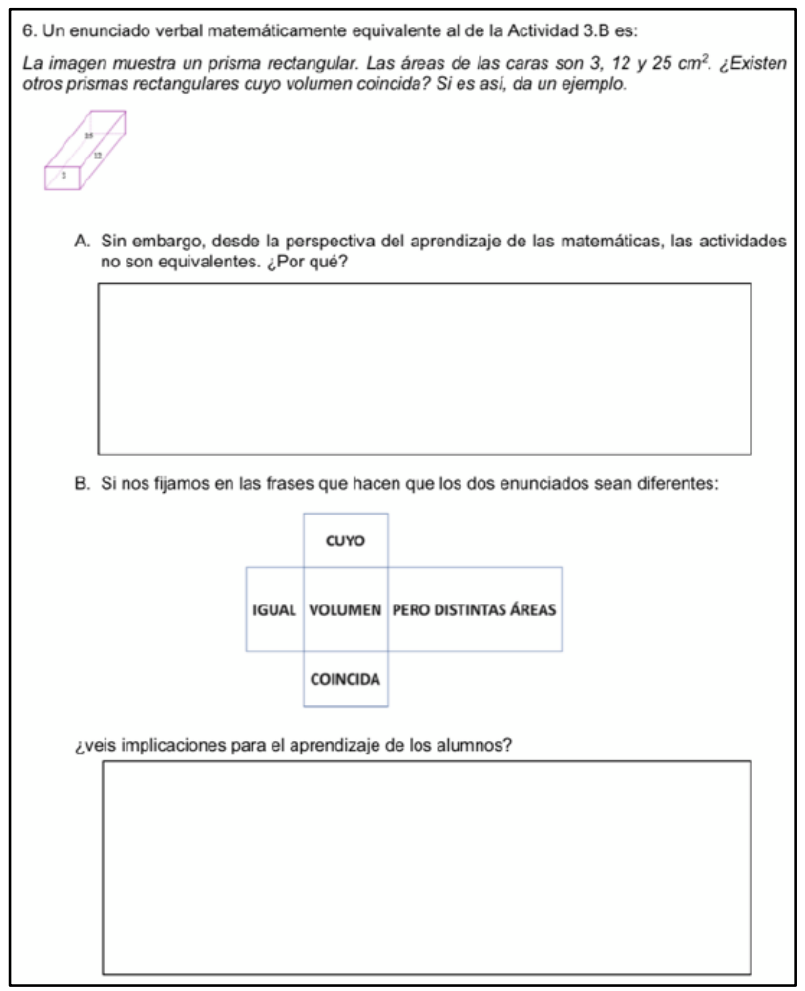

Figura 3. Ítems de la tercera página del cuestionario (continuación)

El segundo grupo participante fueron cuatro estudiantes de tercer curso del Grado que en octubre de 2021 habían empezado la segunda asignatura de educación matemática, 'Gestión e Innovación en el Aula de Matemáticas'. Tres estudiantes habían escrito respuestas ambiguas al cuestionario de 2020, estableciendo distinciones interesantes a primera vista pero sin dar argumentos. La cuarta estudiante había escrito respuestas que indicaban una distinción matemática precisa entre "área plana" y "área lateral o total", a la vez que no había aportado suficiente información para concluir sobre cómo interpretaba la representación de esta distinción en los enunciados del cuestionario donde la palabra "área" no aparecía adjetivada. Tras lograr contactar con todas ellas y explicarles que queríamos indagar en sus respuestas, aceptaron ser entrevistadas.

Las entrevistas tuvieron que adaptarse al formato virtual por las circunstancias de emergencia sanitaria del momento. Cada entrevista se inició compartiendo pantalla para recordar y preguntar sobre algunas respuestas de la futura maestra en el cuestionario escrito del curso anterior. Por ejemplo, si la estudiante había escrito que "el área es el área de un cuadrado o el área de un triángulo" y "el volumen es el volumen de un prisma o el volumen de un cubo", volvíamos sobre estas respuestas a fin de lograr profundizar en la comprensión de los conceptos. En la segunda parte de cada entrevista, se mostraba la situación de la Figura 4 y se dejaba que la estudiante la leyera. La situación permite comparar fragmentos de habla de dos maestras en sus aulas de primaria, manteniendo la especificidad del contenido matemático. Se supone, en particular, que la tarea escogida 
por las maestras garantiza que el contenido de la relación entre área y volumen está presente en el trabajo del aula, a la vez que las preguntas de la entrevista problematizan si este contenido se hace explícito. La situación permaneció en un espacio de la pantalla mientras se conversó en torno a: i) ¿Qué vocabulario matemático usa cada maestra?; ii) ¿Te llama la atención alguna diferencia sobre cómo hablan?; iii) ¿Cómo se refieren a la relación matemática entre área y volumen?; iv) ¿Cuál de las dos opciones de habla es más adecuada si se quiere discutir con los alumnos esta relación y por qué?

Situación. En dos clases de quinto de primaria planificadas para trabajar la relación entre área y volumen, las maestras proyectan en la pizarra digital la siguiente representación de un prisma rectangular con las medidas de alto, largo y ancho:

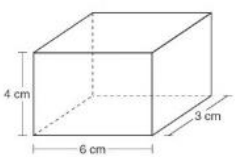

Luego se dirigen a sus grupos de alumnos hablando así:

Maestra 1. Si encontramos las áreas de las caras, ¿podremos saber el volumen del prisma?

Maestra 2. Tenemos las medidas de los lados. Si encontramos las medidas de las caras, ¿podremos saber la medida del prisma?

Figura 4. Situación inicial proporcionada en las entrevistas

\section{Análisis de datos de cuestionario y de entrevista}

El análisis de datos de cuestionario fue iterativo y orientado al estudio de temas literalmente expresados en el texto escrito de las futuras maestras. Aplicamos un análisis del contenido que persiguió codificar las respuestas a las tareas con vistas al logro del objetivo. La unidad de análisis fueron frases compuestas por dos o más palabras gramaticalmente con sentido pudiendo haber más de un verbo. Así, la unidad de análisis es de complejidad lingüística intermedia al no ser una palabra ni tampoco, por lo general, la redacción completa de una respuesta. Para estas frases, indagamos su adecuación con un código de los tipos matemático, didáctico matemático, lingüístico, pedagógico e indeterminado. En la columna con ejemplos de la Tabla 1, entre paréntesis se informa de la estudiante ( $\mathrm{e}_{1} \ldots$ e 51$)$ y de la parte del cuestionario donde se halla la unidad transcrita. Empezamos el procedimiento ubicando frases en los tipos matemático, didáctico matemático y linguí́stico, que fueron los que guiaron el diseño de las tareas del cuestionario. Los tipos pedagógico e indeterminado aparecieron más tarde, para frases que nos pareció que no tenía sentido interpretar en ninguno de los códigos iniciales.

Tabla 1. Códigos organizadores de datos de cuestionario

\begin{tabular}{l|ll}
\multicolumn{1}{c}{ Códigos } & \multicolumn{1}{c}{ Descripción } & \multicolumn{1}{c}{ Ejemplos } \\
\hline Matemático & $\begin{array}{l}\text { Señala hechos y significados } \\
\text { de matemáticas }\end{array}$ & $\begin{array}{l}\text { "el área se asocia a superficies planas de dos } \\
\text { dimensiones" }\left(\mathrm{e}_{2}, 1\right)\end{array}$ \\
$\begin{array}{l}\text { Didáctico } \\
\text { matemático }\end{array}$ & $\begin{array}{l}\text { Señala aspectos de } \\
\text { enseñanza y aprendizaje de } \\
\text { matemáticas }\end{array}$ & $\begin{array}{l}\text { "se pide que encontremos cuál es el volumen a } \\
\text { partir de saber las medidas de las caras" }\left(\mathrm{e}_{6},\right. \\
\text { 5A) }\end{array}$ \\
Lingüístico & $\begin{array}{l}\text { Señala formas léxicas y } \\
\text { gramaticales }\end{array}$ & $\begin{array}{l}\text { "en el enunciado 3A el concepto de área se } \\
\text { explicita" }\left(\mathrm{e}_{1}, 5 \mathrm{~B}\right)\end{array}$ \\
Pedagógico & $\begin{array}{l}\text { Señala aspectos generales de } \\
\text { "la segunda es reflexiva y nos hace pensar más } \\
\text { enseñanza y aprendizaje } \\
\text { allá de la construcción del ejercicio" }(\mathrm{e} 4,4)\end{array}$ \\
Indeterminado & $\begin{array}{l}\text { No responde o es inteligible } \\
\text { "no lo sé" }\left(\mathrm{e}_{18}, 3 \mathrm{~B}\right)\end{array}$
\end{tabular}


A partir de los resultados de este análisis de contenido, se seleccionaron 15 cuestionarios donde el código lingüístico se mostró con una frecuencia de frases superior al promedio. En particular, se identificaron cinco o más frases de contenido lingüístico verbal explícito para estos cuestionarios. Esta frecuencia es relativamente baja, lo cual representa por sí mismo el supuesto de la escasa presencia de reflexiones sobre lengua a lo largo de la educación matemática de las participantes. Este subconjunto de datos codificados como lingüísticos de 15 cuestionarios es el punto de partida de un análisis del discurso escrito para el cual manejamos tres subcódigos, justificados inductivamente con base en el análisis de contenido para la relación entre área y volumen, y que son una revisión de los originales en Caro (2020). La Tabla 2 describe y ejemplifica estos subcódigos: forma léxica, forma gramatical y función matemática. Estos subcódigos también se aplicaron en el análisis de datos de entrevista con las cuatro futuras maestras $\left(\mathrm{E}_{1} \ldots \mathrm{E}_{4}\right)$. A pesar de que la situación recreada para las entrevistas no permite mirar dentro del aula de las dos maestras, pensamos los mismos subcódigos ahora interpretados con el trasfondo de la práctica matemática del aula de primaria.

Tabla 2. Subcódigos organizadores del análisis del discurso

\begin{tabular}{|c|c|c|}
\hline Subcódigo & Descripción & Ejemplos de cuestionario y de entrevista \\
\hline \multirow[t]{2}{*}{$\begin{array}{l}\text { Forma } \\
\text { léxica }\end{array}$} & \multirow{2}{*}{$\begin{array}{l}\text { Discute vocabulario } \\
\text { dentro del registro de } \\
\text { la matemática escolar }\end{array}$} & $\begin{array}{l}\text { "porque no introduce el concepto que nosotros estamos } \\
\text { buscando, es decir, el área" }\left(\mathrm{e}_{12}, 5 \mathrm{~A}\right)\end{array}$ \\
\hline & & $\begin{array}{l}\text { "una maestra lo reduce todo a medidas y no habla del } \\
\text { volumen, pero la otra sí utiliza la palabra volumen" }\left(\mathrm{E}_{2}\right)\end{array}$ \\
\hline \multirow[t]{2}{*}{$\begin{array}{l}\text { Forma } \\
\text { gramatical }\end{array}$} & \multirow[t]{2}{*}{$\begin{array}{l}\text { Discute enunciados } \\
\text { dentro del registro de } \\
\text { la matemática escolar }\end{array}$} & $\begin{array}{l}\text { "en este enunciado no queda claro que es importante el } \\
\text { hecho de que se busca mismo volumen, pero con } \\
\text { diferentes áreas" }\left(\mathrm{e}_{23}, 6 \mathrm{~A}\right)\end{array}$ \\
\hline & & $\begin{array}{l}\text { "una dice las áreas de las caras y la otra dice las medidas } \\
\text { de las caras; es bastante diferente porque el alumno } \\
\text { puede pensar que se están haciendo cosas distintas con } \\
\text { las caras del prisma" }\left(\mathrm{E}_{3}\right)\end{array}$ \\
\hline \multirow[t]{2}{*}{$\begin{array}{l}\text { Función } \\
\text { matemática }\end{array}$} & \multirow{2}{*}{$\begin{array}{l}\text { Discute efectos de } \\
\text { formas en la } \\
\text { producción de } \\
\text { significado } \\
\text { matemático }\end{array}$} & $\begin{array}{l}\text { "en el segundo enunciado el alumno debe deducir que } \\
\text { las áreas deben ser distintas" }\left(\mathrm{e}_{1}, 6 \mathrm{~B}\right)\end{array}$ \\
\hline & & $\begin{array}{l}\text { "al ser una tarea con el dibujo del prisma, está claro que } \\
\text { hay un volumen y no es tan importante que la maestra } \\
\text { no diga volumen ni cómo hable porque el dibujo ya } \\
\left.\text { enseña el volumen" ( } E_{1}\right)\end{array}$ \\
\hline
\end{tabular}

\section{Resultados}

En esta sección resumimos resultados principales del estudio en su conjunto, siendo algunos una refinación de resultados parciales presentados en Caro (2020). Informamos sobre aspectos del conocimiento didáctico matemático de las futuras maestras en cuanto a la enseñanza específica de la relación entre área y volumen, para lo cual aportamos frases de respaldo de respuestas de las futuras maestras en cuestionarios y entrevistas. Aunque no era nuestro objetivo investigar cómo las futuras maestras comprenden los conceptos de área y de volumen, y se preguntó por estos conceptos en el cuestionario para ubicar y preparar las tareas sobre conocimiento didáctico especializado, explicamos aspectos de este conocimiento matemático que no esperábamos encontrar.

\subsection{Débil conocimiento matemático de los conceptos de área y volumen}

Con respecto al conocimiento matemático, las futuras maestras en general sugieren significados matemáticos poco claros y a veces erróneos para los conceptos de área y 
volumen. Esta debilidad se evidencia cuando definen el área como el "tamaño" que ocupa una figura geométrica en el plano (es), o como "la multiplicación de la longitud de los costados de una figura" ( $\mathrm{e}_{25}$ ), o bien cuando escriben que "una superficie siempre tiene base y altura" (e $e_{3}$, utilizando los términos de área y superficie como sinónimos matemáticos (e27). Asimismo, varias participantes definen el volumen como "el conjunto de todas las áreas de una figura geométrica" $\left(\mathrm{e}_{13}\right)$, como la "versión 3D del área" $\left(\mathrm{e}_{25}\right)$, o bien como "la cantidad de masa en el interior de un prisma" ( $\left.\mathrm{e}_{37}\right)$, considerando que "toda figura geométrica tiene como dimensiones el largo, el ancho y la altura" (e36). Más en general todavía, la relación entre área y volumen tiende a interpretarse como un contenido al servicio del aprendizaje de otros contenidos tales como las fórmulas de medida del volumen de cuerpos sólidos como el cilindro o el cono. En una de las entrevistas esta interpretación vuelve a aparecer en la voz de una participante distinta:

$\mathrm{E}_{3}$ : Con la relación entre área y volumen ocurre lo mismo que con las fracciones equivalentes que sirven para luego poder sumar y restar fracciones. Pues eso, la relación entre área y volumen sirve para que podamos tener las fórmulas de los cuerpos con volumen.

Cuando se pregunta acerca de cuestiones de enseñanza y de aprendizaje de la relación entre área y volumen en la escuela de primaria, en tres de las entrevistas las estudiantes responden hablando de contenidos de aritmética elemental, que $\mathrm{E}_{2}$ llama "hechos básicos de la matemática", para ejemplificar sus argumentos sobre la prioridad de tener claros los contenidos matemáticos a enseñar. La falta de especificidad didáctica se observa en frases como "el ejercicio pide más reflexión” ( $\left.e_{27}\right)$, “el ejercicio permite hacer deducciones y trabajar el razonamiento" $\left(\mathrm{e}_{2}\right)$, "el enunciado es más claro y permite hacer la representación mental más rápido" $\left(\mathrm{e}_{12}\right)$, "el segundo ejercicio va más allá y necesita de nuestra imaginación y memoria" (e4), o "el primer ejercicio ayudará más a los alumnos". En el siguiente fragmento de otra de las entrevistas, se observa cómo una pregunta específica de índole didáctica se responde con referencias al conocimiento matemático y al conocimiento pedagógico general.

Núria: Si lo que las dos maestras quieren enseñar es la relación entre área y volumen, ¿es importante que hablen de área y de volumen?

E2: $\quad$ Bueno, está claro que las maestras tienen que saber lo que es el área y lo que es el volumen, y luego tienen que buscar tareas que estén bien.

Núria: Entonces, ¿qué significa buscar tareas que estén bien? ¿Qué te parece la tarea que han buscado estas dos maestras?

E2: $\quad$ Tareas que estén bien, que se entiendan bien y que se hayan explicado.

\subsection{Indicios de atención a cuestiones lingüísticas relativas al contenido}

Cuando se les pregunta directamente, las futuras maestras dan valor a que el vocabulario matemático que representa conceptos que se quieren enseñar aparezca explícito en el enunciado de las tareas seleccionadas para dicha enseñanza. La atención al léxico del registro matemático se observa en respuestas como: "se dice de manera explícita que los datos aportados son las áreas de las caras" (e $\left.e_{1}\right)$, "si no introduces el concepto que tú quieres enseñar, es posible que no sean capaces de encontrarlo" ( $\left.\mathrm{e}_{12}\right)$, "incluir área y volumen en el mismo enunciado ayuda a los alumnos a relacionar estos dos conceptos" ( $\left.\mathrm{e}_{28}\right)$, "no se habla del término matemático área" $\left(\mathrm{e}_{40}\right)$, "en la actividad $3 \mathrm{~A}$ se le da el nombre a este concepto" ( $\left.\mathrm{e}_{41}\right)$, o "se introduce vocabulario específico del ámbito matemático" ( $\left.\mathrm{e}_{42}\right)$. 
Las futuras maestras también tienden a asociar uso de vocabulario matemático con la activación de funciones cognitivas; consideran que la utilización de ciertas palabras apoya los procesos de pensamiento matemático del alumno de primaria sin señalar, en sus razonamientos, el contexto de uso de esas palabras ni la relevancia de la tarea matemática. Esto se evidencia en frases como: "un vocabulario más exacto para el alumnado puede ayudar a su comprensión y, por tanto, a la resolución del problema" (e23), "el lenguaje de la segunda no es tan técnico como el de la primera" (e35), "el uso de un vocabulario poco preciso dificulta el entendimiento del ejercicio y la comprensión de éste" (e37), "si las palabras son muy técnicas aumenta la distracción" (e43), o "la implicación de determinadas palabras indirectamente dificulta que el enunciado les sea accesible a primera vista y que acabe siendo más difícil resolverlo de lo que realmente es" (e46). Los adjetivos que se atribuyen al vocabulario sugieren una función que el vocabulario por sí solo no tiene en la lengua, excepto cuando se ubica en una construcción gramatical con funciones de explicación o de argumentación.

Incluso cuando se les pregunta directamente, las futuras maestras no atienden a cambios gramaticales en enunciados de tareas matemáticas ni por tanto discuten posibles efectos de estos cambios en el aprendizaje matemático del alumnado de primaria. Lo anterior se evidencia en las escasas respuestas del tipo: "En este enunciado no queda claro que es importante el hecho de que se busca mismo volumen pero con diferentes área" (e 23$)$, "en el segundo enunciado hay menos información explícita, es necesario que el alumnado piense más" (e 35 ), "el enunciado del ejercicio 3B se encuentra muy explícito y por esta razón los niños podrán sacar un buen aprendizaje" (e42), "un enunciado es más explicativo y se entiende mejor, así que enfocas la atención y no tienes que resolver pequeñas cuestiones durante la lectura" (e43). La atención al vocabulario especializado centra los comentarios sobre el uso de la lengua con apenas referencias a la relevancia de las construcciones gramaticales en la creación de significado matemático y al potencial de unas construcciones por delante de otras.

El siguiente fragmento de entrevista ilustra una estudiante que alude a la presencia explícita de los términos de área, volumen, lados, caras, vértices, forma, prisma, base y cuadrado, que es vocabulario que asocia a la resolución del problema de la Figura 4. Esta estudiante valora la calidad del habla de las maestras del siguiente modo:

Núria: Entonces, ¿qué modo de hablar te parece más convincente si se busca que los alumnos aprendan la relación entre área y volumen?

$\mathrm{E}_{3}$ : $\quad$ A ver, las dos maestras hablan matemáticamente, pero una habla de área y de volumen y la otra no.

Núria: ¿Esta diferencia es importante para lo que se quiere enseñar?

$\mathrm{E}_{3}$ : $\quad$ Lo sería si no hubiera el dibujo del prisma.

Núria: ¿A qué te refieres?

$\mathrm{E}_{3}$ : $\quad$ Si hay un dibujo como el del prisma, donde se ve que hay volumen y áreas, lo que el alumno va a aprender depende menos de cómo hable la maestra.

Núria: ¿Y si no hubiera ese dibujo?

$\mathrm{E}_{3}$ : $\quad$ Pues la maestra tendría que hablar más para dar más detalles y que los alumnos imaginaran bien la situación.

Núria: ¿Qué tipo de detalles tendría que explicar?

$\mathrm{E}_{3}$ : $\quad$ Pues antes de hablar de lados, caras, vértices, volumen ... tendría que explicar un poco la forma del prisma, si la base es un cuadrado y cosas así. 
Tanto en los cuestionarios como en la situación para la entrevista (ver Figuras 2, 3 y 4) había representaciones gráficas en el plano de prismas. Las respuestas escritas de las participantes no se refirieron, sin embargo, a la modalidad gráfica no verbal, sobre la cual nosotras tampoco preguntamos en el cuestionario. En las entrevistas seguimos sin preguntar sobre la lengua no verbal. No obstante, en dos de ellas las estudiantes dan función matemática al dibujo del prisma y relacionan esto con la interpretación de la calidad de las lenguas matemáticas de las maestras en la enseñanza. Las dos estudiantes suponen que los alumnos de primaria tienen la oportunidad de comprender significados matemáticos para los conceptos de área y volumen y para la relación entre ellos mediante la visualización de representaciones particulares del concepto de prisma. Estas representaciones gráficas son un recurso potencial en la enseñanza y el aprendizaje de la relación entre área y volumen, pero no suplen la falta de concreción en ciertas lenguas matemáticas utilizadas para enseñar este contenido en la escuela.

\section{Discusión y conclusiones}

Los resultados de este estudio exploratorio son útiles a dos niveles: por un lado, para saber más acerca del conocimiento matemático y didáctico matemático de las futuras maestras y, por otro lado, para saber más acerca de nuestras prácticas y contenidos en la formación inicial de profesorado de matemáticas. En nuestro trabajo como educadores matemáticos en la formación universitaria del profesorado hay precisamente dos grandes elementos, en términos de Shulman (1986a, 1986b), el conocimiento del contenido de la materia (en nuestro caso, la materia de educación matemática) y el conocimiento del contenido pedagógico (en nuestro caso, la pedagogía para enseñar a enseñar la matemática escolar). Por supuesto, las ideas que tengamos sobre qué es y para qué sirve la educación matemática serán de influencia en los contenidos que seleccionemos y enfaticemos para el trabajo con los estudiantes para profesor. Incluso cuando tengamos escaso margen de decisión en la selección de contenidos (i.e. en el contenido de la materia), nuestras ideas serán de influencia en los modos que seleccionemos de enseñar (i.e. en el contenido de la pedagogía) mediante las formas en las que interaccionemos con las estudiantes y mediante cómo presentemos y justifiquemos las tareas en el aula universitaria. Siempre incidimos necesariamente en el contenido de la pedagogía porque tenemos posicionamientos sobre cómo las personas aprenden (ya sea matemáticas o a enseñar matemáticas). Si son posicionamientos basados en premisas socioculturales, entonces recurriremos a pedagogías centradas en la interacción con y la mediación de los otros y, aquí, la lengua que utilicemos para comunicarnos será esencial.

Sean cuales sean las premisas que sustentan las distintas pedagogías para enseñar a enseñar matemáticas, y las distintas teorías del aprendizaje humano que nos guíen, los resultados de este estudio son útiles en la consideración del conocimiento del contenido de la educación matemática a trabajar con las futuras maestras. Para empezar, los datos revelan un conocimiento matemático débil de los conceptos de área y volumen y de la relación entre ellos. Es posible que esto se deba a no haber trabajado suficientemente en el pasado tareas sobre las habituales falsas relaciones de dependencia entre área y volumen (e.g. a mayor área, mayor volumen; a menor área, menor volumen) o a otros fenómenos como los mencionados en Clements et al. (2018). Al ser preguntadas sobre estos conceptos la mayoría de las participantes tuvieron dificultades para definir o explicar área y volumen, esto es, para usar la lengua matemáticamente en la elaboración de definiciones o explicaciones. Hay errores en las respuestas, pero sobre todo hay explicaciones incompletas o demasiados generales que incluyen más de un concepto matemático (e.g. volumen y capacidad; área y plano). En cualquier caso, no parece 
razonable hablar en términos de capacidades y pensar que las estudiantes no han sido capaces de construir estos conceptos; seguramente no han tenido oportunidades suficientes de participar en contextos en los que discutir e interactuar en torno a significados matemáticos para área y volumen. Si bien no esperábamos que las futuras maestras tuvieran conocimientos didácticos relativos al uso de la lengua en la enseñanza de matemáticas, entendíamos que habían superado las etapas educativas donde se enseñan los conceptos de área y volumen y su relación. De manera ingenua dábamos por supuesto que los contextos -con sus tareas y sus participantes- para discutir y compartir los significados matemáticos de estos conceptos se habrían habilitado $\mathrm{y}$, a partir de ahí, unos significados se habrían construido y otros se habrían revisado.

Las respuestas pedagógicas generalistas con apenas alusiones a cuestiones particulares de contenidos de la matemática escolar, de su enseñanza y aprendizaje, son otro resultado emergente de nuestra investigación. Algunas de las participantes se fijan en el vocabulario matemático en el enunciado de tareas valorando que sea explícito, pero no atienden a los cambios gramaticales en los enunciados ni en los posibles efectos de estos cambios en el aprendizaje matemático del alumnado de primaria. Estos resultados apuntalan la conveniencia de formar al profesorado de matemáticas en conocimiento matemático, didáctico y lingüístico sobre registros matemáticos específicos en torno a distintos contenidos curriculares. La lengua profesional experta que las futuras maestras deberán utilizar en su práctica de aula ha de promover el aprendizaje conceptual de los alumnos por lo que es del todo necesario nombrar los conceptos y explicarlos poniéndolos en relación con y nombrando sus cualidades y otros conceptos, tal como ya señaló Pimm (2019/1987). Construcciones gramaticales como [prismas rectangulares] "cuyo volumen coincida" o "con igual volumen pero diferentes áreas" generan oportunidades distintas de comprensión de la relación entre volumen y área, no solo por el vocabulario específico sino también por las cláusulas que funcionan como productores de conexiones lógicas. Si un objeto de la enseñanza es esta relación, la segunda construcción, que contrasta igual volumen con diferente área, es relevante.

No queremos acabar sin discutir por qué un artículo como este es relevante en un monográfico sobre investigación en educación matemática que aspira a comprender cómo los aspectos sociales y culturales impactan en los procesos de enseñanza y de aprendizaje de matemáticas. El profesorado de matemáticas necesita saber que las lenguas matemáticas que utilice en clase condicionarán la creación y distribución de oportunidades de aprendizaje matemático entre los alumnos; esta es una reflexión a la vez ampliable al grupo de formadores de profesorado de matemáticas (White, Crespo, \& Civil, 2016). El uso que hagamos de las lenguas matemáticas en el aula universitaria o en los entornos de desarrollo profesional y los modos en los que enseñemos a reflexionar a las futuras maestras y al profesorado en activo sobre el papel de la lengua en la educación matemática condicionarán la creación y distribución de oportunidades de enseñanza de contenidos matemáticos en la escuela. En aquellas escuelas donde no llegan recursos materiales como libros de texto y tecnologías digitales, o donde no llegan para todos los alumnos y familias, debemos garantizar aún con mayor convicción y eficacia que no se pierdan las oportunidades de enseñanza y de aprendizaje que brinda el recurso inmaterial de la lengua matemática experta de la maestra.

\section{Agradecimientos}

Agencia Nacional de Investigación y Desarrollo / Beca Chile Magíster Profesionales de la Educación 77200019. Proyecto MICINN España, PID2019-104964GB-I00. 


\section{Referencias}

Ball, D. L., Thames, M, H., \& Phelps, G. (2008). Content knowledge for teaching: What makes it special? Journal of Teacher Education, 59(5), 389-407.

Caro, A. (2020). Conocimiento de futuros profesores de primaria sobre el uso de la lengua para la enseñanza de matemáticas: Estudio con la relación área-volumen. Trabajo de Fin de Máster. Universitat Autònoma de Barcelona, https://ddd.uab.cat/ record/234594

Chitera, N. (2011). Language of learning and teaching in schools: An issue for research in mathematics teacher education? Journal of Mathematics Teacher Education, 14(3), 231-246.

Clements, D. H., Sarama, J., Van Dine, D. W., Barret, J. E., Cullen, C. J. ..., \& Earnes, C. L. (2018). Evaluation of three interventions teaching area measurement as spatial structuring to young children. The Journal of Mathematical Behavior, 50, $23-41$.

De Gamboa, G., Badillo, E., \& Ribeiro, C. M. (2015). El horizonte matemático en el conocimiento para la enseñanza del profesor: Geometría y medida en educación primaria. PNA, 10(1), 1-24.

Departament d'Educació (2013). Competències bàsiques de l'àmbit matemàtic. Barcelona: Generalitat de Catalunya.

Erath, K., Ingram, J., Moschkovich, J., \& Prediger, S. (2021). Designing and enacting instruction that enhances language for mathematics learning. A review of the state of development and research. ZDM-Mathematics Education, 53, 245-262.

Essien, A., Chitera, N., \& Planas, N. (2016). Language diversity in mathematics teacher education: Challenges acrossuevathree countries. En R. Barwell et al. (Eds.), Mathematics education and language diversity. The 21 ${ }^{\text {st }}$ ICMI Study (pp. 103-119). Nueva York: Springer.

Gee, J. P. (1999). An introduction to discourse analysis: Theory and method. Nueva York: Routledge.

Ingram, J. (2018). Moving forward with ethnomethodological approaches to analysing mathematics classroom interactions. ZDM-Mathematics Education, 50, 1065-1075.

Pimm, D. (2019/1987). Speaking mathematically: Communication in mathematics classrooms. Londres, Inglaterra: Routledge Revivals.

Planas, N. (2018). Language as resource: A key notion for understanding the complexity of mathematics learning. Educational Studies in Mathematics, 98(3), 215-229.

Planas, N. (2021). How specific can language as resource become for the teaching of algebraic concepts? ZDM-Mathematics Education, 53, 277-288.

Planas, N., Badillo, E., \& Chico, J. (2019). Soportes lingüísticos matemáticamente relevantes en la enseñanza de la ecuación cuadrática. En J. M. Marbán, M. Arce, A. Maroto, J. M. Muñoz-Escolano, \& À. Alsina (Eds.), Actas del XXIII Simposio de la Sociedad Española de Investigación en Educación Matemática (pp. 493-502). Valladolid: SEIEM.

Planas, N., Chico, J., García-Honrado, I., \& Arnal-Bailera, A. (2020). Discursos del alumno y del profesor en clase de matemáticas. En E. Badillo, N. Climent, C. Fernández, \& M. T. González (Eds.), Investigación sobre el profesor de matemáticas: 
Práctica de aula, conocimiento, competencia y desarrollo profesional (pp. 19-41). Salamanca: Ediciones de la Universidad.

Planas, N., Morgan, C., \& Schütte, M. (2018). Mathematics and language. Lessons and directions from two decades of research. En T. Dreyfus, M. Artigue, D. Potari, S. Prediger, \& K. Ruthven (Eds.), Developing research in mathematics education. Twenty years of communication, cooperation and collaboration in Europe (pp. 196210). Londres, Inglaterra: Routledge.

Planas, N., Morgan, C. y Schütte, M. (2021). Developing a perspective on multiplicity in the study of language in mathematics classrooms. En N. Planas, C. Morgan, \& M. Schütte (Eds.), Classroom research on mathematics and language: Seeing learners and teachers differently (pp. 3-21). Londres, Inglaterra: Routledge.

Prediger, S. (2019). Investigating and promoting teachers' expertise for languageresponsive mathematics teaching. Mathematical Education Research Journal, 31, 367-392.

Prediger S., Erath K., \& Moser-Opitz, E. (2019). The language dimension of mathematical difficulties. En A. Fritz, V. Haase, \& P. Räsänen (Eds), International handbook of mathematical learning difficulties (pp. 437-455). Cham, Suiza: Springer.

Radford, L. \& Barwell, R. (2016). Language in mathematics education research. En Á. Gutiérrez, G. C. Leder, \& P. Boero (Eds.), The second handbook of research on the psychology of mathematics education. The journey continues (pp. 275-313). Rotterdam, Holanda: Sense Publishers (Brill).

Rangnes, T., \& Meaney, T. (2021). Preservice teachers learning from teaching mathematics in multilingual classrooms. En N. Planas, C. Morgan, \& M. Schütte (Eds.), Classroom research on mathematics and language: Seeing learners and teachers differently (pp. 201-218). Londres, Inglaterra: Routledge.

Shulman, L. S. (1986a). Those who understand: Knowledge growth in teaching. Educational Researcher, 15(2), 4-14.

Shulman, L. S. (1986b). Paradigms and research programs in the study of teaching: A contemporary perspective. En M. Wittrock (Ed.), Handbook of research on teaching (pp. 3-36). Nueva York: Macmillan

Shulman, L. S. (1987). Knowledge and teaching: Foundations of the new reform. Harvard Educational Review, 57(1), 1-23.

Shulman, L. S. (2005). Signatures pedagogies in the professions. Daedalus -Journal of the American Academy of Arts \& Sciences, Summer no., 52-59.

White, D. Y., Crespo, S., \& Civil, M. (Eds.) (2016). Cases for mathematics teacher educators: Facilitating conversations about inequities in mathematics classrooms. Charlotte, NC: Information Age Publishing.

\section{Referencias de las autoras}

Abigail Caro, Universitat Autònoma de Barcelona (España). abigail.caro@ecampus.uab.cat

Núria Planas, Universitat Autònoma de Barcelona (España). Nuria.Planas@uab.cat 


\title{
Exploratory study with preservice primary teachers on mathematical languages for teaching the relationship between area and volume
}

\author{
Abigail Caro, Universitat Autònoma de Barcelona \\ Núria Planas, Universitat Autònoma de Barcelona
}

In this article, we summarise an exploratory study on the interpretation of mathematically relevant uses of language in a setting of mathematics education preservice training in Barcelona. We take a sociocultural view on mathematics teaching and learning and mathematics teacher education in our approach to the preparation of teachers of mathematics to improve their languages in the school classroom for the enhancement of conceptual mathematics learning. Based on our knowledge of the research literature on mathematics education and language, we believe that mathematics teacher preparation programs would benefit from the deliberate and research-oriented inclusion of contents of language use within the registers of school mathematics. The study at present was conducted in the curricular context of the Primary Education Degree at our University, with a relatively small body of student teachers. By means of deductive analytical methods applied to responses from a questionnaire, we first examined evidences of what primary school student teachers know or anticipate about the function of language in mathematics teaching and learning. We then added inductive methods of discourse analysis applied to data from the questionnaire responses amplified through selective interviews for the exploration of what the student teachers know or anticipate when the teaching content is the relationship between area and volume. Our results show participants with weak understanding of the concepts of area and volume, as well as in-construction views of the language as resource oriented to supporting conceptual mathematics learning in the school. Interviews particularly served to illustrate the extent to which the student teachers perceive language use in the classroom with school learners from a pedagogically general standpoint. They tend to reduce the mathematical languages for content-specific teaching and learning (e.g. around the relationship between area and volume) to issues of vocabulary proficiency rather than to developing grammars of explaining and relating concepts. 